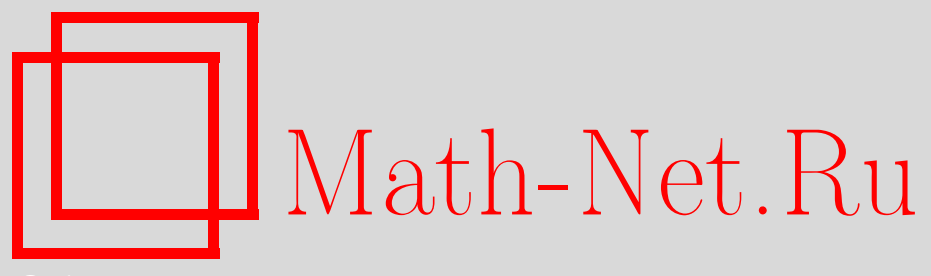

Е. М. Скориков, Точные значения информационных колмогоровских поперечников классов Соболева в одномерном случае, Матем. заметки, 2009, том 86, выпуск $1,154-156$

DOI: https://doi.org/10.4213/mzm8364

Использование Общероссийского математического портала Math-Net.Ru подразумевает, что вы прочитали и согласны с пользовательским соглашением http://www . mathnet.ru/rus/agreement

Параметры загрузки:

IP: 3.85 .73 .92

26 апреля 2023 г., 14:19:21

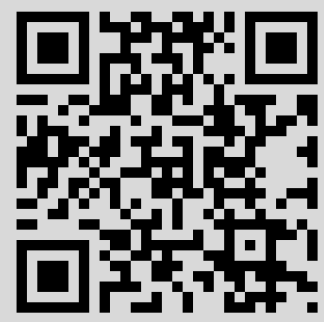




\section{Точные значения информационных колмогоровских поперечников классов Соболева в одномерном случае}

\section{Е. М. Скориков}

Везде далее $X, Y$ - банаховы пространства, $M$ - непустое центрально симметричное относительно нуля подмножество $X, \mathscr{B}(X, Y)$ - множество всех линейных ограниченных операторов из $X$ в $Y, B_{X}$ - замкнутый единичный шар $X$. Пусть $L_{m} \subset X$ - подпространство размерности $m, V^{n} \subset X$ - подпространство коразмерности $n$.

Величины

$$
\begin{aligned}
& d_{m}(M, X) \stackrel{\text { def }}{=} \inf _{L_{m} \subset X} \sup _{x \in M} \inf _{y \in L_{m}}\|x-y\|_{X}, \\
& \lambda_{n}(M, X) \stackrel{\text { def }}{=} \inf _{L_{n} \subset X, A \in \mathscr{B}\left(X, L_{n}\right)} \sup _{x \in M}\|x-A x\|_{X},
\end{aligned}
$$

называются колмогоровским и линейным поперечниками множества $M$ соответственно.

ОПРЕДЕЛЕНИЕ. Информационным колмогоровским поперечником назовем величину

$$
d_{m}^{n}(M, X) \stackrel{\text { def }}{=} \inf _{V^{n}} \sup _{b \in X} d_{m}\left(M \cap\left(V^{n}+b\right), X\right) .
$$

В работе [1] показано, что для произвольного множества $M \subset X$ справедливо неравенство

$$
d_{m}^{n}(M, X) \leqslant \lambda_{n+m}(M, X)
$$

Доказательство основной теоремы базируется на аппарате приближения функции сплайнами (см. [2]).

Обозначим через $\widetilde{\Gamma}_{2 k, r}$ множество 1-периодических идеальных сплайнов порядка $r$, у которых $r$-я производная имеет на периоде не более, чем $2 k$ существенных перемен знака, а через $\varphi_{2 k, r} \in \widetilde{\Gamma}_{2 k, r}-$ Эйлеров идеальный сплайн. Пусть $\mathbb{T}^{1}=\mathbb{R} / \mathbb{Z}$ - одномерный тор, $\widetilde{L}_{q}=\widetilde{L}_{q}\left(\mathbb{T}^{1}\right)-$ пространство Лебега.

Множество функций

$$
\widetilde{W}_{\infty}^{r} \stackrel{\text { def }}{=}\left\{f \in A C^{(r-1)}\left(\mathbb{T}^{1}\right):\left\|f^{(r)}\right\|_{\infty} \leqslant 1\right\}
$$

называется классом Соболева.

Для доказательства основной теоремы, нам потребуется

Теорема 1 ([2; с. 260]). Для произволъных $k \in \mathbb{N}, q \in[1, \infty]$ справедливы равенства

$$
d_{2 k}\left(\widetilde{W}_{\infty}^{r}, \widetilde{L}_{q}\right)=\lambda_{2 k}\left(\widetilde{W}_{\infty}^{r}, \widetilde{L}_{q}\right)=\left\|\varphi_{2 k, r}\right\|_{q}
$$

Справедлива

Теорема 2. Пусть $n+m=2 k$ четно; тогда выполнено равенство

$$
d_{m}^{n}\left(\widetilde{W}_{\infty}^{r}, \widetilde{L}_{q}\right)=d_{n+m}\left(\widetilde{W}_{\infty}^{r}, \widetilde{L}_{q}\right) .
$$

ДоказАтельство. Оценка сверху вытекает из (1) и (2). Для оценки снизу достаточно оценить величину

$$
\inf _{V^{n}} d_{m}\left(\widetilde{W}_{\infty}^{r} \cap V^{n}, \widetilde{L}_{q}\right),
$$

для чего достаточно показать справедливость следующей леммы.

(C) Е. М. Скориков, 2009 
Лемма 1. Для произвольных подпространств $V^{n}, L_{m} \subset X$, обладающих одним из условий

(*) $1 \in L_{m}$,

$(* *) \quad 1 \in X \backslash V^{n}$,

существует функция $f_{*} \in \widetilde{\Gamma}_{n+m, r}$ такая, что

$$
f_{*} \in V^{n}, \quad \inf _{l_{m} \in L_{m}}\left\|f_{*}-l_{m}\right\|_{q}=\left\|f_{*}\right\|_{q} .
$$

ДокАЗАТЕЛЬСтво леммы полностью аналогично доказательству леммы 6.1 .5 [2; с. 255]. Пусть $V^{n}=\bigcap_{s=1}^{n} \operatorname{ker}\left\langle\cdot, y_{s}^{*}\right\rangle$. Так же, как и в лемме 6.1.5, для произвольного вектора $\xi$ на единичной сфере $S^{2 k}$ пространства $l_{1}^{2 k+1}$ определяется функция $g_{0}(\xi, t)$, непрерывно и нечетно зависящая от $\xi$;

$$
\begin{gathered}
\tau_{0} \stackrel{\text { def }}{=} 0, \quad \tau_{s} \stackrel{\text { def }}{=} \sum_{i=1}^{s}\left|\xi_{s}\right|, \quad s=1, \ldots, 2 k+1, \\
g_{0}\left(\xi, \tau_{s}\right) \stackrel{\text { def }}{=} 0, \quad g_{0}(\xi, t) \stackrel{\text { def }}{=} \operatorname{sgn} \xi_{s}, \quad \tau_{s-1}<t<\tau_{s}, \quad s=1, \ldots, 2 k+1 .
\end{gathered}
$$

Далее, аналогично строятся функции $g_{j}(\xi, t)$ :

$$
g_{j}(\xi, t) \stackrel{\text { def }}{=} \int_{0}^{t} g_{j-1}(\xi, u) d u+c_{j}(\xi), \quad j=1, \ldots, r,
$$

где для $j=1, \ldots, r-1$ константы $c_{j}(\xi)$ определяются условием $\int_{0}^{1} g_{j}(\xi, t) d t=0, c_{r}(\xi)=0$. Положим

$$
f(\xi, t)=g_{r}(\xi, t)+c(\xi) .
$$

В случае $(*) c(\xi)$ определяется условием $c(\xi) \stackrel{\text { def }}{=} \arg \inf _{\lambda}\left\|g_{r}(\xi, \cdot)-\lambda\right\|_{q}$.

Для случая $(* *)$ можно без ограничения общности считать, что $\left\langle 1, y_{1}^{*}\right\rangle \neq 0$; тогда константа $c(\xi)$ определяется условием $\left\langle f(\xi, \cdot), y_{1}^{*}\right\rangle=0$.

Аналогично строится непрерывное и нечетное поле векторов

$$
\eta: S^{2 k} \rightarrow \mathbb{R}^{2 k}
$$

Зададим $\eta_{1}(\xi)=\int_{0}^{1} g_{0}(\xi, t) d t$. В случае $(*)$, представляя $L_{m}=\operatorname{Lin}\left\{1, e_{2}, \ldots, e_{m}\right\}$, положим

$$
\begin{aligned}
\eta_{i}(\xi) & =\int_{0}^{1} e_{i}(t)|f(\xi, t)|^{q-1} \operatorname{sgn} f(\xi, t) d t, & & i=2, \ldots, m, \\
\eta_{i+n}(\xi) & =\left\langle f(\xi, \cdot), y_{i}^{*}\right\rangle, & & i=1, \ldots, n .
\end{aligned}
$$

Для случая $(* *)$ определим

$$
\begin{aligned}
\eta_{i+1}(\xi) & =\int_{0}^{1} e_{i}(t)|f(\xi, t)|^{q-1} \operatorname{sgn} f(\xi, t) d t, & & i=1, \ldots, n \\
\eta_{i+n}(\xi) & =\left\langle f(\xi, \cdot), y_{i}^{*}\right\rangle, & & i=2, \ldots, n .
\end{aligned}
$$

В силу теоремы Борсука существует вектор $\xi^{*} \in S^{2 k}$, для которого $\eta\left(\xi^{*}\right)=0$. Это, во-первых, означает, что $f_{*}=f\left(\xi^{*}, \cdot\right) \in V^{n}$ и, во-вторых, из соотношений двойственности,

$$
\left\|f_{*}\right\|_{q}=\inf _{l_{m} \in L_{m}}\left\|f_{*}-l_{m}\right\|_{q}
$$

В случае $1 \leqslant q<\infty$ лемма доказана. Доказательство в случае $q=\infty$ следует из аналогичных лемме 6.1 .5 [2; с. 255] рассуждений.

Автор выражает благодарность И. Г. Царькову, А. С. Кочурову за внимание к работе и полезные замечания. 


\section{СПИСОК ЦИТИРОВАННОЙ ЛИТЕРАТУРЫ}

[1] Е. М. Скориков, Изв. РАН. Сер. матем., 71:3 (2007), 173-196. [2] Н. П. Корнейчук, Сплайны в теории приближения, Наука, М., 1984.

\section{Е. М. Скориков}

Московский государственный университет им. М. В. Ломоносова

E-mail: skorikov_eugene@mail.ru
Поступило

20.11.2008 